\title{
ANALISIS MULTITEMPORAL DEL CAMBIO DE COBERTURA VEGETAL DE LA MICROCUENCA EL VOLCÁN A PARTIR DE LA COMPRA DE ÁREAS ESTRATÉGICAS.
}

\section{MULTITEMPORAL ANALYSIS OF THE CHANGE OF VEGETATION COVERAGE OF THE VOLCAN MICROBASIN FROM THE PURCHASE OF STRATEGIC AREAS.}

\author{
PEÑALOZA, L. K.' CARVAJAL, F. A. ${ }^{2}$, HERNANDEZ, N. J. ${ }^{3}$ \\ 'Ing. Leidy Karina Peñaloza Miranda, Ingeniera Ambiental, \\ e-mail: karina.penaloza@outlook.es
}

${ }^{2}$ Msc Fidel Antonio Carvajal S. Profesor Titular, Programa de Ingeniería Ambiental, Facultad de Ingenierías y Arquitectura, Universidad de Pamplona, Tel: 5685303 (ext. 140) e-mail: fidelcarvajal@unipamplona.edu.co

${ }^{3}$ Msc. Nathalie Johana Hernández Pérez. Profesor Asociado, Programa de Economía, Facultad de Ciencias Económicas y Empresariales, Universidad de

Pamplona, Tel: 5685303 (ext. 140) e-mail: nahepe@hotmail.com

\section{Entidad}

Ciudadela Universitaria. Universidad de Pamplona, Norte de Santander, Colombia

Tel: 57-7-5685303, Fax: 57-7-5685303 Ext. 140

\author{
http://www.unipamplona.edu.co/
}

\section{Resumen}

El objetivo de la presente investigación fue determinar los cambios de la cobertura vegetal entre los años 1992 a 2017 de la microcuenca El Volcán, Pamplona, Norte de Santander, para determinar los cambios de las coberturas antes y después de la compra de áreas estratégicas. Para esto, se seleccionaron y procesaron imágenes satelitales de 1992, 1997, 2014 y 2017 correspondientes a los años definidos; luego, se aplicó la metodología Corine Land Cover a escala 1:100000 adaptada para Colombia. El procesamiento de la información se realizó mediante el software ArcGIS con una escala 1:25000. Los resultados revelaron una recuperación positiva de las áreas de protección, mientras que la microcuenca presenta una variación entorno a la cobertura vegetal, con influencia de los sistemas productivos en áreas boscosas, áreas con procesos de recuperación y disminución de otras coberturas.

Palabras clave: Corine Land Cover, Multitemporal, servicios ecosistémicos

Abstract 
The objective of this research was to determine the changes in of vegetation coverage 1992 and 2017 in the El Volcán microbasin, Pamplona, Norte de Santander. For this purpose, satellite images from 1992, 1997, 2014 and 2017 were selected and processed for the defined years to determine the changes in coverage before and after the purchase of strategic areas; The Corine Land Cover methodology was then applied on a 1:100000 scale adapted for Colombia. The information was processed using ArcGIS software on a scale of $1: 25000$. The results revealed a positive recovery of the protected areas, while the microbasin presents a variation around the vegetation cover, with influence of the productive systems in forested areas, areas with recovery processes and decrease of other coverages.

Keywords: Corine Land Cover, Multitemporal, ecosystem services

\section{INTRODUCCIÓN}

La cuenca hidrográfica es un sistema constituido por elementos bióticos y abióticos que interrelacionados ofrecen una serie de servicios ecosistémicos, determinantes para el desarrollo sostenible de cualquier sociedad (Carmona y Guzman, 2015; Rodríguez, 2017). La sobreexplotación y el uso irracional de los recursos naturales derivado de actividades como el crecimiento demográfico, la expansión de la frontera agrícola, la tala indiscriminada y la sobreutilización del recurso hídrico generan una de las amenazas más grandes hacia estos ecosistemas, modificando las coberturas vegetales, las condiciones del suelo y consecuentemente sus servicios ecosistémicos; servicios que son reconocidos como el puente de unión entre la biodiversidad y el ser humano (Ministerio Ambiente y Desarrollo Sostenible, 2012; Herrera \& Granadillo, 2015).

Por tanto, las autoridades ambientales y los entes territoriales han buscado alternativas para contrarrestar los efectos generados por las actividades antrópicas, mediante la implementación de áreas de protección, delimitando espacios geográficos claramente definidos, reconocidos, dedicados y gestionados por medios legales $u$ otros tipos de medios eficaces para conseguir la conservación a largo plazo de la naturaleza, sus servicios ecosistémicos y sus valores culturales asociados (Dudley, 2008; Vera, 2017)

Este estudio se enfocó en evaluar los cambios de la cobertura vegetal en la microcuenca El Volcán, a partir de la adquisición de la reserva El Volcán, localizada en jurisdicción de las veredas Alto Grande y El Totumo del municipio de Pamplona Norte de Santander (Angulo et al, 2017). Para esto, se recopiló información secundaria y primaria, referente imágenes satelitales de la zona de estudio, usos del suelo, verificación en campo, entre otros; posteriormente se realizó el respectivo análisis y discusión de las variaciones multitemporales y la relación de la dinámica social con el ecosistema, finalmente se presentan las conclusiones y recomendaciones para futuras investigaciones.

\section{METODOLOGIA}

La metodología de esta investigación es de tipo cuantitativo con enfoque descriptivo, se plantea un análisis multitemporal de la cobertura vegetal de la microcuenca EI Volcán a partir de la compra de áreas estratégicas, ubicadas en la parte alta de la microcuenca El Volcán. 


\subsection{ANÁLISIS MULTITEMPORAL DE LA COBERTURA VEGETAL}

Se aplicó la metodología Corine Land Cover escala 1:100.000 adaptada para Colombia, priorizando la clase y la cobertura de tierra hasta nivel tres de interpretación. Esta fase abarcó la selección y adquisición de imágenes satelitales de los años 1992, 1997, 2014 y 2017, años definidos para de determinar los cambios de las coberturas antes y después de la compra de áreas estratégicas.

\subsection{PROCESAMIENTO DE LA INFORMACIÓN}

Para la sistematización y análisis se empleó el software ArcGIS a una escala 1:25.000; así como la verificación en campo para la evaluación de las coberturas. De igual forma, se generó una tabla multitemporal de cambios, en donde se presentan las transiciones que se producen entre los periodos 1992 y 2017, lo que permite observar, no sólo las zonas de cambio sino también comparar la cobertura original y la actual, calculando las áreas para cada tipo de cobertura.

Finalmente, se establece una relación de los cambios de cobertura con las actividades antropogénicas, especialmente la agricultura y eventos puntuales ocurridos a lo largo del tiempo como la adquisición de áreas estratégicas.

\section{ANALISIS $Y$ DISCUSIÓN DE RESULTADOS}

\subsection{LOCALIZACIÓN DE LA ZONA DE ESTUDIO}

La microcuenca el Volcán, se encuentra ubicada al sur oriente del municipio de Pamplona, Norte de Santander, Colombia, comprende un área aproximada de 1.079,68 Has, un perímetro de $17.25 \mathrm{Km}$ y una longitud axial de 5,6 Km (Fernández, Suarez y Pérez, 2007), así mismo, su rango altitudinal oscila entre 2830 y 3600 m.s.n.m., está integrada por las veredas $\mathrm{El}$ Rosal, Alto Grande, Totumo y parte del barrio Zulia; cuyas aguas fluyen hacia el Noreste para conformar junto con la Quebrada Monteadentro el río Pamplonita a los 2.340 m.s.n.m (Mapa 1) (Ramírez, Fernández y Solano, 2005; Galarza, 2016).

Mapa 1. Ubicación geográfica microcuenca el

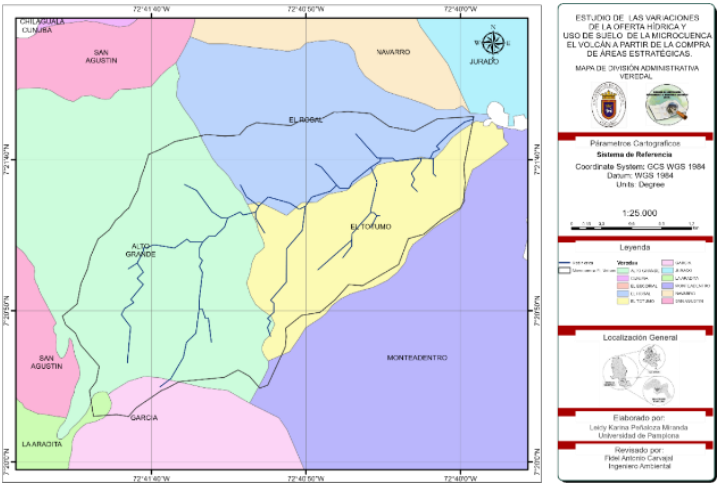

Volcán, Pamplona Norte de Santander

Fuente: Autor, 2017

En su recorrido de $6.35 \mathrm{Km}$ la microcuenca El Volcán tiene como afluentes principales la Quebrada Los Monos, Quebrada La Despensita, Quebrada El Pedregal, Quebrada Chinchipa, Quebrada El Salto, Quebrada Las Peñas, el Chorro de Buena Vista y Chorro El Zanjón del Mortiño. (Carvajal, 2017). El Volcán crea una red hidrográfica con diversidad de paisajes y condiciones topográficas y climatológicas típicas de la zona alta andina (Sánchez, Gelvis y Solano, 2007; Villamizar \& Justinico, 2017).

Inmersa en dicha microcuenca se encuentra el área estratégica El Volcán ubicada en las veredas el Totumo y Alto Grande (Mapa 2), con una extensión de aproximadamente 603,5 Has adquiridas con la financiación de CORPONOR, con la finalidad de su conservación, protección y recuperación (Corporación Autónoma Regional de Norte de Santander, 2005) su rango altitudinal oscila entre los 3000 y 3500 m, incluyendo 
las zonas de vida Altoandina y páramo (Sánchez et al., 2007).

Mapa 2. Reserva El Volcán.

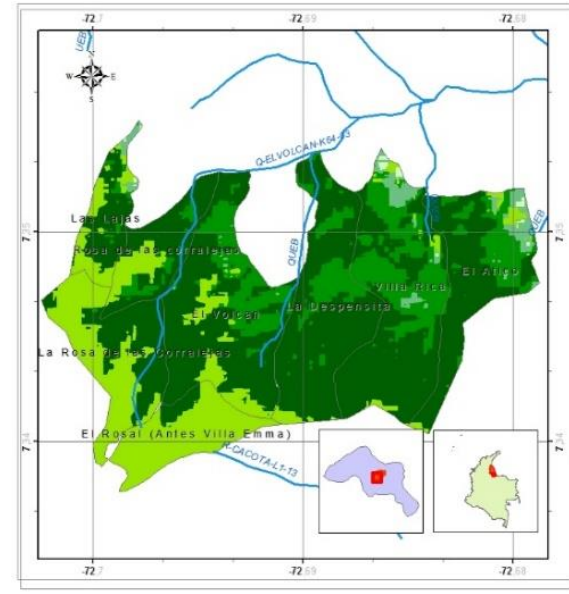

Fuente: Autor, 2017

La reserva es el punto de nacimiento para la quebrada Los Monos y La Despensita entre otros afluentes que proveen su recurso para el municipio de Pamplona y a su vez se unen a otras aguas para conformar el denominado Río Pamplonita (PBOT Pamplona, 2015).

\subsection{ANÁLISIS MULTITEMPORAL DE LA COBERTURA VEGETAL EN LA MICROCUENCA EL VOLCÁN}

En primer lugar, en la microcuenca El Volcán se identificaron dos clases de cobertura: Territorios agrícolas (Clase 2.) y Bosques y áreas seminaturales (Clase 3). Los Territorios agrícolas presentaron dos coberturas de nivel tres, Mosaico de pastos y cultivos con código 2.4.2 y Mosaicos de pastos con espacios naturales (2.4.4.), a su vez, los Bosques y áreas seminaturales registraron, tres coberturas de nivel tres, Bosque fragmentado con código 3.1.3., Arbustal (3.2.2) y vegetación secundaria (3.2.3.). Cabe aclarar que la metodología Corine Land Cover está sujeta a interpretación de la persona que la aplique, presentando estas variaciones en las comparaciones realizadas.
Para el año 1992, fueron identificadas dos tipos de coberturas nivel uno, donde a su vez se subdividen hasta nivel tres; la cobertura con mayor área de ocupación son bosques y áreas seminaturales con 462,656 Ha equivalente al $62,61 \%$, correspondiente a coberturas de bosques fragmentados, arbustales y vegetación secundaria 0 en transición (Mapa 3).

De este modo, podría afirmarse que para el año 1992, las zonas demarcadas con esta cobertura se caracterizan por comprender territorios cubiertos por vegetación arbustiva desarrolladas de forma natural en diferentes densidades y sustratos, así mismo se encuentra cubierta por bosques fragmentados que representan los bosques naturales densos 0 abiertos cuya continuidad es afectada por otros tipos de coberturas como pasto, 0 cultivos y vegetación secundaria o en transición que se presenta luego de la intervención o destrucción de la vegetación primaria.

Mapa 3. Clasificación cobertura vegetal 1992
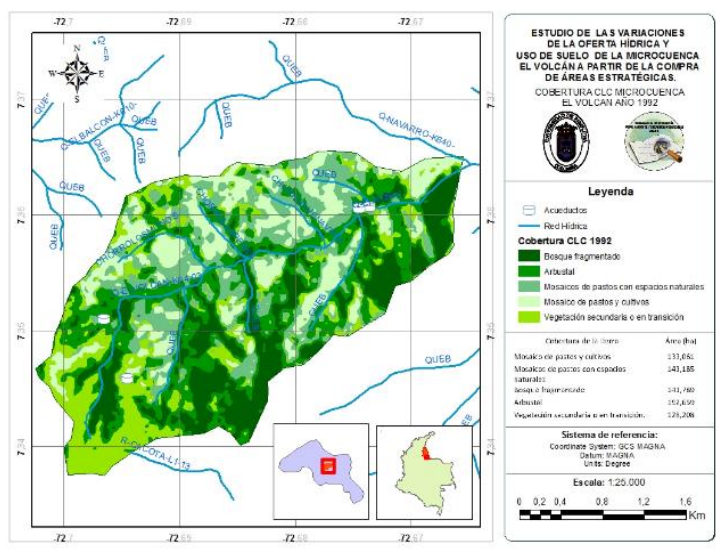

Fuente: Autor, 2017

En menor proporción, aunque igualmente significativa, se identifican coberturas de tipo agrícola heterogénea, donde se observan coberturas de tipo mosaico de pastos y cultivos, mosaicos de pastos con espacios naturales, ocupando un área de 133,061 Ha y 143,185 Ha respectivamente, 
las cuales equivalen a un $37,39 \%$ de la extensión de territorio de estudio.

Al igual que en el año 1992, en 1997 la cobertura "Arbustales" se distingue por ser la vegetación dominante, presenta disminución en su área en un $2,4 \%$ equivalente al 17,85 $\mathrm{Ha}$, dando paso a la expansión de la vegetación secundaria y bosques fragmentados en $4,73 \%$ y $2,1 \%$ respectivamente (Mapa 4).

Mapa 4. Clasificación cobertura vegetal 1997.

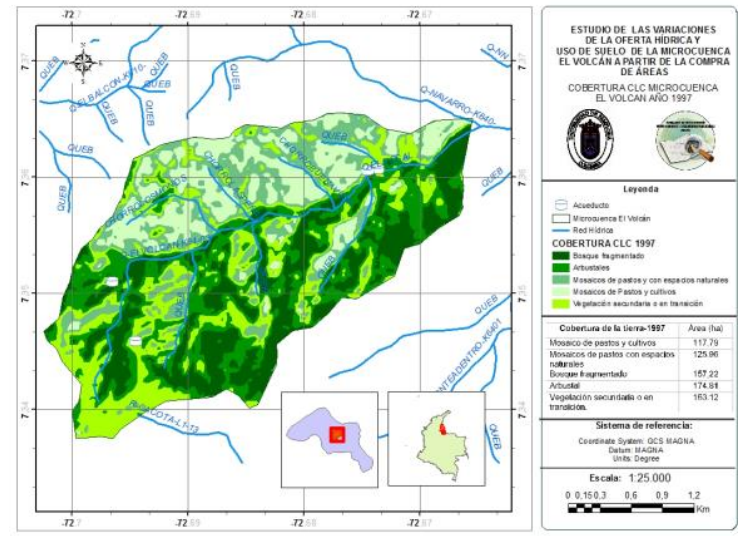

Fuente: Autor, 2017

Se presenta una recuperación de la cobertura vegetal en las veredas Alto Grande y Totumo, mientras que la vereda El Rosal, expande constantemente su área agrícola y ganadera, ocasionando la pérdida de bosques fragmentados que a su vez podrían catalogarse como riparios al encontrarse alrededor de los tributarios, y cierto porcentaje de vegetación secundaria o en transición. Las demás coberturas identificadas presentan variaciones respecto a la fecha anterior exhibiendo una disminución en su extensión en 4,4\%, equivalente a $32,5 \mathrm{Ha}$.

Para el año 2005, por parte de las autoridades ambientales de la Región, se inicia un proceso de recuperación de las fuentes abastecedoras del casco urbano, se crea e instaura el Plan de Manejo Ambiental de la microcuenca El Volcán en el municipio de Pamplona.
En el año 2014 coberturas como mosaico de pastos y cultivos, mosaicos de pastos con espacios naturales y bosques fragmentados presentan un aumento su área de expansión en un 0,57\%, 2,48\%, $7,09 \%$ respectivamente, caso opuesto a las coberturas de arbustales y vegetación secundaria que disminuyen en un $2,25 \%$ y $7,91 \%$ respecto al año 1997 (Mapa 5). Cabe resaltar que dicha variación de cobertura se acentúa en la vereda El Rosal, donde los bosques primarios, vegetación secundaria y arbustales, se encuentran deteriorados casi en su totalidad, dejando descubiertos nacientes, tributarios y pequeñas quebradas abastecedoras.

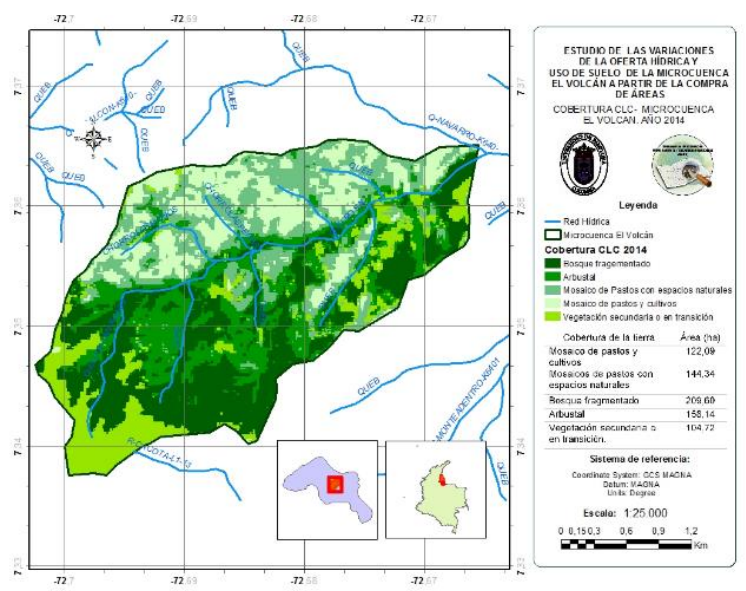

Mapa 5. Clasificación cobertura vegetal 2014.

Fuente: Autor, 2017

En el Mapa 6 se observa la clasificación de la cobertura vegetal, junto con la ubicación de las viviendas a lo largo de la microcuenca, georreferenciados previamente. En este caso, el área de estudio presenta una recuperación considerable, respecto al año de 1992, donde la cobertura vegetal de bosques ha sido la más beneficiada, presentando un $11,79 \%$ de recuperación, esto debido a la implementación de áreas de protección y recuperación de zonas de vida. 
Al realizar un análisis comparativo de las coberturas para el periodo 1992-2017, (Tabla 1) se identifica un cambio muy positivo para el ecosistema y sus servicios, puesto que, se realiza la identificación de bosque de fragmentación, con un área de ocupación de $228,9 \mathrm{Ha}$, del cual se logra recuperar un $11,79 \%$ equivalente a 87,12 $\mathrm{Ha}$, respecto al año inicial de estudio. Esta cobertura es un signo de recuperación del bosque natural y de protección de los cuerpos de agua presentes en el área de estudio.

Mapa 6. Clasificación cobertura vegetal 2017.
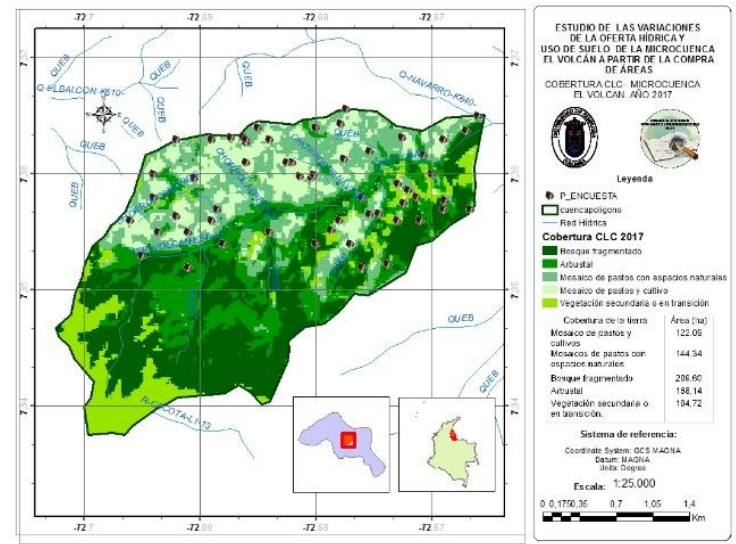

Fuente: Autor, 2017

Tabla 1. Análisis comparativo de los cambios generados en la cobertura vegetal, periodo

\begin{tabular}{|c|c|c|c|c|c|c|}
\hline $\begin{array}{l}\text { I } \\
\text { I } \\
0\end{array}$ & ن & 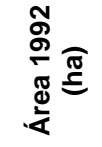 & $0^{\circ}$ & 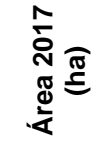 & ১0 & $\begin{array}{l}\text { o } \\
\stackrel{0}{8} \\
\frac{0}{2}\end{array}$ \\
\hline \multirow{2}{*}{2} & 2.4 .2 & 133,06 & 18,01 & 111,93 & 15,15 & $-2,86$ \\
\hline & 2.4.4. & 143,19 & 19,38 & 150,86 & 20,42 & 1,04 \\
\hline \multirow{3}{*}{3} & 3.1.3. & 141,79 & 19,19 & 228,91 & 30,98 & 11,79 \\
\hline & 3.2.2. & 192,65 & 26,07 & 155,33 & 21,02 & $-5,05$ \\
\hline & 3.2.3. & 128,21 & 17,35 & 91,86 & 12,43 & $-4,92$ \\
\hline & Cotal & 738,9 & 100,0 & 738,9 & 100,0 & 0,0 \\
\hline \multicolumn{4}{|c|}{ Aumenta } & \multicolumn{3}{|c|}{ Disminuye } \\
\hline
\end{tabular}

Fuente: Autor, 2017

Posteriormente, se presenta una disminución de la cobertura de arbustales y vegetación secundaria o en transición del $5,05 \%$ y $4,92 \%$ respectivamente, la cual fue reemplazada por la cobertura anteriormente mencionada, se determina que para el año 1992 existían $462 \mathrm{Ha}$ de coberturas de bosque y áreas seminaturales, mientras que para el 2017 se obtuvieron 476,1 Ha, dando como resultado un aumento de cobertura vegetal de $14,1 \mathrm{Ha}$ correspondientes al $2 \%$ del territorio y una tasa de recuperación de vegetación anual de 0,08\%; valores que resultan poco significativos para un periodo de recuperación de 20 años, lo cual resalta que la recuperación de las zonas de protección es positiva, pero el territorio aledaño carece de control y normatividad, lo cual afecta al territorio y sus servicios ecosistémicos.

Analizando directamente los cambios efectuados en la reserva El volcán, siete de los ocho predios adquiridos y que actualmente la conforman, presentaron un porcentaje mayor de bosques fragmentados respecto al año de estudio inicial, así mismo, los territorios de producción agrícola tienden a 0 para el año 2017, aumentando la cobertura de bosque y áreas seminaturales, lo cual representa a nivel general una recuperación de $27,86 \%$ de la cobertura principal y la reducción casi total de los mosaicos de pastos y cultivos aun registrados en la zona.

Según Torres, Murcia y Sánchez (2014), la Reserva El Volcán contienen un ecosistema heterogéneo de matorrales, predominando el estrato arbustivo y abundancia de epifitas vasculares, dichas características permiten recuperar especies de fauna y el aumento del recurso hídrico, concorde a lo expresado, Zarta, Rivera y Sánchez (2015) plantean una diversidad alta de especies florales y un aumento de cobertura vegetal, caracterizado por un clímax de especies, presentando mayor cantidad de características propias de un bosque primario.

En general, la microcuenca El Volcán contempla un cambio positivo para el ecosistema; sin embargo, presenta el 
aumento de territorios agrícolas, y mosaicos de pastos y cultivos, evidenciando afectación de la vegetación primaria principalmente en la vereda el Rosal debido a la expansión de la frontera agrícola, llegando a una disminución considerable de sus bosques y arbustales, desprotegiendo nacimientos y quebradas representativos de la zona, de acuerdo a Ortega (2016), quien evalúa la ecología principal de la cuenca del río Pamplonita, entendida como el conjunto de los sistemas naturales que forman parte de la infraestructura del capital ambiental soporte para garantizar la protección y conservación de la biodiversidad, la prestación de servicios ecosistémicos y satisfacer las necesidades básicas de la población, estudio que evidencia una situación crítica de este aspecto para el municipio de Pamplona, lo cual indica la incorporación de esta zona en proyectos de recuperación, restauración y rehabilitación de manera que se garantice en el mediano y largo plazo la prestación adecuada de los servicios ecosistémicos.

Tal como lo plantea Espinel, Rivera y Castellanos (2016) es importante emplear planes de manejo en pro de los recursos, crear una conciencia sobre la importancia del recurso suelo logrando la adopción por parte de la comunidad de prácticas mucho más amigables y adecuadas sobre el medio.

\section{CONCLUSIONES}

La microcuenca el Volcán representa uno de los principales motores para el desarrollo económico y poblacional; constituye un punto cardinal para los servicios de abastecimiento de la población pamplonesa, la cual provee tanto al $40 \%$ de la cabecera municipal de dicha región, como a la comunidad asentada en la zona, el recurso hídrico presenta usos de tipo, pecuario, agrícola e incluyendo servicios públicos, donde los dos últimos representan el mayor porcentaje de utilidad en un 44,57 y 54,83 $\%$ respectivamente.
Para el periodo comprendido entre 1992 y 2017, la microcuenca el volcán muestra un cambio positivo para ambiente y sus servicios ecosistémicos, demostrando ganancias en su vegetación primaria; las coberturas de bosque y áreas seminaturales para el 2017 presentan una extensión de 476,1 $\mathrm{Ha}$; dando como resultado un aumento de cobertura vegetal de $14,1 \mathrm{Ha}$ respecto a 1992, correspondientes al $2 \%$ del territorio y una tasa de recuperación de vegetación anual de 0,08 \%, mientras que los cambios presentados a lo largo del área estratégica presentan una tasa de recuperación anual del $4 \%$, dichos valores resultan poco significativos para un periodo de recuperación de 20 años, resalta que la recuperación de las zonas de protección es positiva, pero el territorio aledaño carece de control y normatividad, lo cual afecta al territorio y los servicios prestados por dicha microcuenca.

La producción agrícola se cataloga como la economía principal de la zona rural, donde la vereda el rosal se caracteriza por ser un punto importante para la agricultura, esta vereda presenta variaciones en su producción a lo largo del tiempo, así mismo la densidad poblacional ha sufrido cambios constantes evidenciando un mayor número de residentes en dicha vereda respecto a las otras ubicadas en la microcuenca; donde la compra de áreas de protección puede ser un factor de cambio en esta variable, generando migración desde los predios adquiridos a predios aledaños a la zona, lo cual ocasiona un impacto en la cobertura vegetal, al realizar solo un cambio de locación.

La dinámica poblacional de la microcuenca ha sufrido cambios constantes que afectan notablemente los servicios del ecosistema; para el año 1993-2005, existió un aumento considerable de la población rural, lo cual se evidencia al analizar los datos del DANE, esto retrasa la recuperación del terreno y aumenta la extracción del recurso hídrico, lo 
mencionado anteriormente, resalta la importancia de conocer la dinámica social y económica de la zona a la cual se quiere intervenir.

El éxito de proyectos de restauración para la recuperación de los servicios ecosistémicos dependerá de un cambio ligado al componente social y económico de la zona, no solo del área adquirida sino de sus alrededores, aquellos que interactúen directamente con los recursos que se desean recuperar, así mismo los componentes de conocimiento y educación juegan un papel fundamental en el proceso de recuperación de dicha zona.

\section{REFERENCIAS BIBLIOGRÁFICAS}

Angulo, W. J., Mendoza, J. A. y Uriel, H. U. (2017). Análisis de la vulnerabilidad por fenómenos de remoción en masa en la Cuenca Tanauca estudio de caso. Revista Ambiental Agua, Aire y Suelo. ISSN 1900-9178. Volumen (8), Numero (2). DOI: https://doi.org/10.24054/19009 178.v2.n2.2017.3276

Carvajal, G. (2017). Entrevista realizada al personal de EMPOPAMPLONA del municipio de Pamplona, Norte de Santander.

Carmona, J y Guzmán S. (2015). La Ciudad-Cuenca: una expresión socioecológica del territorio. Bitácora Urbano Territorial, Volumen 26, Número 1, p. 103-110, 2016. ISSN electrónico 2027-145X. ISSN impreso 0124-7913. http://dx.doi.org/10.15446/bitacora.v 26n1.40254

CORPONOR. Corporación Autónoma Regional de Norte de Santander. (2005) Proyecto de distribución de caudales dentro del proceso de reglamentación del uso del agua en las microcuencas el volcán y Monteadentro a partir de sus nacimientos en su paso por las veredas el totumo, alto grande, el rosal, navarro, jurado, barrio el Zulia y Monteadentro del municipio de Pamplona hasta su confluencia con la cuenca del rio pamplonita.

Dudley, N. (Editor) (2008). Directrices para la aplicación de las categorías de gestión de áreas protegidas. Gland, Suiza: UICN. $x+96 p p$.

Espinel, R. M., Rivera, H. U. y Castellanos, C. (2016). Plan de manejo ambiental para los proyectos de extracción de material de arrastre, localizados en las microcuencas quebradas volcán y navarro del municipio de Pamplona, Norte de Santander. Revista Ambiental Agua, Aire y Suelo. ISSN 1900-9178. Volumen (7), Numero (1). DOI: https://doi.org/10.24054/19009178.v 1.n1.2016.3282

Fernández, N., Suarez, C. y Pérez, A. (2007). Modelación y simulación dinámica para la gestión de caudales en la cuenca Alta del Río Pamplonita- Un Balance Hídrico de Consumo. Universidad de Pamplona.

Galarza, R. (2016). Construcción de una variable aleatoria para simular el movimiento de caudales medios en el Rio Fonce (Santander) en el marco de la axiomatica de Andrey Kolmogorov. Revista Ambiental Agua, Aire y Suelo. ISSN 19009178. volumen (7), Numero (2). DOI: https://doi.org/10.24054/19009 178.v2.n2.2016.3264

Gutierrez, T., Castellanos, C. y Hernández, N. (2016). El ordenamiento territorial frente a las consecuencias de los cambios climáticos. Revista Ambiental Agua, Aire y Suelo. ISSN 1900-9178. Volumen (7), Numero (2).

DOI: https://doi.org/10.24054/190091 78.v2.n2.2016.3338

Herrera, J. D. y Granadillo, J. A. (2015). Control de erosión y establecimiento de cobertura vegetal en $49 \mathrm{~m} 2$ de suelo degradado en el efecto de 
borde del bosque seco tropical de la UFPS Ocaña. Revista Ambiental Agua, Aire y Suelo. Volumen (6), Numero

DOI: https://doi.org/10.24054/19009

178.v1.n1.2015.3242

IDEAM. (2010). Leyenda Nacional de Coberturas de la Tierra. Metodología CORINE Land Cover adaptada para Colombia Escala 1:100.000. Instituto de Hidrología, Meteorología y Estudios Ambientales. Bogotá, D.C., 72p.

Ivanova, Y. y Sarmiento, A. (2014). Evaluación de la huella hídrica de la ciudad de Bogotá como una herramienta de la gestión del agua en el territorio urbano. Revista Ambiental Agua, Aire y Suelo. ISSN 1900-9178. Volumen (4), Numero (2),

1-5.

DOI: https://doi.org/10.24054/190091 78.v2.n2.2013.427

Melo, J., Saavedra, S. y Ramón, J. A. (2017). Evaluación de la adsorcion de $C U+2$ y azul de metileno en biosorbentes de bajo costo obtenidos a partir de biomasa residual de la agroindustria de cítricos. Revista Ambiental Agua, Aire y Suelo. ISSN 1900-9178. Volumen (8), Numero (2). DOI: https://doi.org/10.24054/190091 78.v2.n2.2017.3277

Meneses, V. B., Álzate, D. y Mosquera, J. (2016). Sistema de optimización de las técnicas de planificación en agricultura de precisión por medio de drones. Revista Ambiental Agua, Aire y Suelo. ISSN 1900-9178. Volumen (7), Numero (2). DOI: https://doi.org/10.24054/190091 78.v2.n2.2016.3268

Ministerio de Ambiente y Desarrollo Sostenible. (2012). Política Nacional de Gestión Integral de Biodiversidad y Servicios Ecosistémicos. Bogotá

Moreno, C. y Rueda, L. (2016). La educación ambiental como herramienta para la recuperación de la cobertura vegetal, mediante prácticas agro-ecológicas en la comunidad minera asograstorres, asociación de gravilleros de Sabana de Torres. Revista Ambiental Agua, Aire y Suelo. ISSN 1900-9178. Volumen (7), Numero (1). DOI: https://doi.org/10.24054/190091 78.v1.n1.2016.3260

Ortega, J. Y. (2016). Estructura ecológica principal de la cuenca del Río Pamplonita. Revista Ambiental Agua, Aire y Suelo. ISSN 19009178. Volumen (7), Numero (1). DOI: https://doi.org/10.24054/19009178.v 1.n1.2016.3258

Plan Básico de Ordenamiento territorial PBO Pamplona (2015). Alcaldía de Pamplona, Pamplona, Norte de Santander, Colombia.

Ramírez, A., Fernández, N. y Solano, F. (2005) Dinámica fisicoquímica y calidad del agua en la microcuenca el volcán, en el municipio de Pamplona, Colombia. Universidad de Pamplona.

Rodríguez, Y. A. (2017). Conservación de humedales en el marco de gestión de cuencas hidrográficas. Puerto Rondón-Arauca. Revista Ambiental Agua, Aire y Suelo. ISSN 19009178. Volumen (8), Numero (2). DOI: https://doi.org/10.24054/19009 178.v2.n2.2017.3281

Sánchez, L., Gelviz, S. y Solano, F. (2007). Plantas con Flores de la Reserva el Volcán, Vereda Alto Grande Pamplona, Norte de Santander, Colombia. Universidad de Pamplona. Revista Bistua.

Torres, S., Murcia, M. A. y Sánchez, L. R. (2014). Composición floristica y estructura del matorral de la franja Altoandina en la cuenca del Río Pamplonita. Revista Ambiental Agua, Aire y Suelo. ISSN 19009178. Volumen (4), Numero (2), 8898. DOI: https://doi.org/10.24054/19009178.v 
2.n2.2013.435

Zarta, H., Rivera, M. y Sánchez, R. (2015) Estado actual de las áreas estratégicas del municipio de Pamplona, Norte de SantanderColombia. Universidad de Pamplona.

Vera, J. A. (2017). El compromiso ambiental de la alta dirección en la adopción de sistemas de gestión ambiental ISO 14001. Revista Ambiental Agua, Aire y Suelo. ISSN 1900-9178. Volumen (8), Numero (2).

DOI: https://doi.org/10.24054/19009 178.v2.n2.2017.3283

Villamizar, V. A. y Justinico, A. J. (2017). Reconstrucción paleoclimatica y paleoambiental de los territorios de la llanura inundable del araucana a partir del análisis de sedimentos recientes, Departamento de Arauca. Revista Ambiental Agua, Aire y Suelo. ISSN 1900-9178. Volumen (8), Numero

DOI: https://doi.org/10.24054/19009 178.v1.n1.2017.3272 